\title{
Método aplicado en la disminución de accidentes de tránsito en el cantón Loja
}

\section{Method applied in the reduction of traffic accidents in the canton of Loja}

\author{
Wilson Jaramillo Sangurima \\ Verónica Alexandra Muñoz Sotomayor \\ Universidad Internacional del Ecuador, Ecuador
}

Autor por Correspondencia: jaramillowilson@gmail.com, valex.munoz@gmail.com Fecha de recepción: 30 de Enero de 2017 - Fecha de aceptación: 25 de Marzo de 2017

Resumen: En el área urbana y rural perteneciente al Cantón Loja en el transcurrir del año 2014, ocurrieron 1716 accidentes de tránsito, el $96 \%$ por causa humana, generándose 31 fallecidos y 386 personas heridas. Lejos de pensar que estos accidentes fueron acontecimientos fortuitos, se ha comprobado que los accidentes de tránsito son consecuencia de un fallo evitable y hasta cierto punto predecible, lo que genera la necesidad de combatir este problema ejecutando actividades fundamentadas en un método científico, como es el caso particular del método denominado "Investigación - Acción". El método de investigación comprende el análisis de las causas y estrategias aplicadas a los patrones de ocurrencia recolectados entre los años de estudio, durante los cuales, las acciones de prevención se ejecutaron en función de los resultados de las estadísticas de los años anteriores. Como resultado del trabajo ejecutado en el cantón Loja, se logró una reducción de accidentes de tránsito, presentándose en el transcurso de año 2015 un total de 944 accidentes y de 526 en el año 2016, disminuyéndose los índices de mortalidad y morbilidad, y evaluando las estrategias de acción en función de las causas predominantes.

Palabras clave: accidentes de tránsito; reducción de accidentes; seguridad vial; investigación acción

Abstract: In urban and rural areas of Loja, in 2014, 1716 traffic accidents occurred, 96\% by human cause, generating 31 deaths and 386 people injured. Many people might think that these incidents were unavoidable and unpredictable events, however, it has been show that traffic accidents are the result of an avoidable failure and to some extent predictable, which generates the need to combat this problem by running founded on a scientific method activities, as the case of the method called "Research - Action". The research method comprises the analysis of the causes and strategies applied to the patterns of occurrence collected between the years of study, during which, the prevention actions were executed according to the results of the statistics of previous years. As a result of the work carried out in Loja, a reduction of traffic accidents was achieved, with a total of 944 accidents occurring in 2015 and 526 accidents in 2016, decreasing mortality and morbidity rates, this shows the effectiveness of the method called action research and evaluates the strategies of action according to the predominant causes.

Key words: traffic accidents; reducing accidents; road safety; action research 


\section{Introducción}

La organización Mundial de la Salud OMS define a los accidentes de tránsito como un problema de salud pública; se estima que "1.2 millones de personas pierden la vida cada año en todo el mundo a causa de choques en la vía pública” (Jorge et al. 2010), esta situación además, constituye un fenómeno complejo, por la cantidad de factores involucrados en su ocurrencia que abarca a sectores sociales, culturales, económicos y normativos; cada una de estas perspectivas aborda a esta problemática como causante y consecuente, demostrando una alta influencia de los niveles de educación de conductores y peatones, imposición y cumplimiento de normativas, desarrollo económico y conducta social (Norza et al. 2014).

Como problema de salud, la OMS establece que para el año 2020, los accidentes de tránsito serán la tercera causa de muerte alrededor del mundo; sin embargo a diferencia de otras enfermedades que afectan a la población como el cáncer, enfermedades cardiovasculares o el SIDA; los accidentes de tránsito pueden tener un mayor nivel de evitabilidad, ya que al involucrar al factor humano se cuestiona su ocurrencia por caso fortuito y la atribución misma al término "accidente".

Según Tomas (2015) nada en cuanto le sucede al individuo a lo largo de su existencia se debe sólo al azar. Incluso los hechos más incomprensible para nuestra inteligencia obedecen a la causalidad, ya pueda ser develada en algún momento, ya permanezca oculta para nuestra comprensión. El accidente siempre ha estado rodeado de una imagen de azar, de generación espontánea e imprevisión implícitas en su propia definición. Sería igualmente irreal creer que los accidentes son cosa del destino o fruto de la causalidad y que, por consiguiente, están fuera de nuestro control y no podemos hacer nada para evitarlos.

Frente a tales concepciones los especialistas en este fenómeno descubrieron ya hace mucho tiempo que los accidentes no son en absoluto acontecimientos fortuitos, inevitables, impredecibles y dependientes de la suerte, sino que por el contrario de la inmensa mayoría de los casos siguen parámetros característicos de distribución; es decir, el accidente globalmente es siempre una consecuencia de algún fallo evitable y hasta cierto punto predecible del sistema (Montoro y Toledo, 1997).

Para el caso de Ecuador, la planificación, regulación y control del transporte terrestre, tránsito y seguridad vial urbana, son competencia exclusiva de cada municipio, de acuerdo a principios de descentralización; establecido en el numeral 6 del art. 264 de la Constitución de la República del Ecuador, el art. 55 del Código Orgánico de Organización Territorial, Autonomías y Descentralización COOTAD, el primer párrafo del art. 30.4 de la Ley Orgánica de Transporte Terrestre, Tránsito y Seguridad Vial, la Resolución Nro. 006 CNC-2012 del Consejo Nacional de Competencias y la Resolución Nro. 025 - DE - ANT - 2013 de fecha 26 de abril de 2013.

Con las competencias exclusiva bajo su responsabilidad, a finales del mes de agosto de 2013 el Municipio de Loja a través de agentes civiles llamados también policías municipales de tránsito, inicia los controles en la vía pública, recabando estadísticas sobre la ocurrencia de accidentes de tránsito, siendo así, que según los datos de la Unidad Municipal de Transporte Terrestre Tránsito y Seguridad Vial de Loja "UMTTTSV”, entre la última semana del mes de 
agosto hasta el 31 de diciembre del 2013 se registraron 573 accidentes de tránsito, 12 fallecidos y 97 personas heridas, 93\% ocurrido en el área urbana y 7\% en el área rural. Así mismo, durante el año 2014 con un registro de matriculación de 42543 vehículos se reporta un total de 1716 accidentes, 31 fallecidos y 386 personas heridas, $91 \%$ en el área rural y 9\% en el área urbana.

De estas estadísticas, se obtiene que para el cantón Loja, el 96\% de las causas de accidentes de tránsito se producen por causa humana y el resto se atribuye al factor climático, falla mecánica o mal estado de la vía. De esta forma, además de ser un problema de salud pública, los accidentes de tránsito también son un problema social y económico, ya que no se puede menospreciar los enormes costos que se derivan del gasto de un accidente. Según la Unidad Municipal de Transporte Terrestre Tránsito y Seguridad Vial de Loja (UMTTTSV) en el año 2014 el perjuicio económico por daños materiales causados por accidentes de tránsito supera los 3.5 millones de dólares, sin tomar en cuenta los costos que soporta la sociedad por bajas laborales, incapacidad laboral, absentismo, pérdida de productividad, asistencia de salud en los hospitales o clínicas, tramitaciones, peritajes, o la atención de todo tipo de secuelas como invalidez, orfandad, viudedad, entre otros.

En base a lo expuesto se evidencia la necesidad de combatir la ocurrencia de accidentes de tránsito, aplicando como caso de estudio el cantón Loja, emprendiendo en actividades que respondan a métodos científicos que permitan resolver este problema, como es el caso particular del método de "Investigación - Acción", el cual según Hernández (2010), pretende, esencialmente, propiciar el cambio social, transformar la realidad y que las personas tomen conciencia de su papel en ese proceso de transformación; también señala, que este método, representa el estudio de un contexto social donde mediante un proceso de investigación con pasos "en espiral", se investiga al mismo tiempo que se interviene, lo que permite plantear la siguiente pregunta de investigación:

- ¿Cómo se puede disminuir para el año 2015 y 2016, un $20 \%$ la ocurrencia de accidentes de tránsito, tomando como base de referencia lo sucedido en el año 2014 en el cantón Loja, aplicando el método Investigación - Acción?

De acuerdo a las posibilidades de la metodología a emplear se evalúa la efectividad de estrategias para la disminución de accidentes de tránsito, referidas principalmente al factor humano, comprobando la hipótesis que la aplicación del método de investigación acción permite en el transcurso del año 2015 y 2016, disminuir en un 20\% el número de accidentes de tránsito, en relación a lo ocurridos en el año 2014 en el cantón Loja.

\section{Materiales y Métodos}

Para realizar la investigación se determinó como ambiente de trabajo el Municipio de Loja a través de la UMTTTSV, la cual tiene como tarea planificar, regular y controlar el transporte terrestre, tránsito y seguridad vial en el cantón Loja.

El cantón Loja se encuentra ubicado al sur del Ecuador; comprende 6 parroquias urbanas y 13 parroquias rurales, con un total de población de 214.855 habitantes (según el censo del 2010) de los cuales 180.617 pertenecen al área urbana. 
Con el propósito de responder a la pregunta de investigación y cumplir con el objetivo del estudio, se empleó un enfoque de investigación de tipo cualitativo y el diseño de investigación denominado según Hernández (2010) “investigación -acción”, el cual considera una visión técnico científica, cuyo modelo consiste en un conjunto de decisiones en espiral, las cuales se basan en ciclos repetidos de análisis para conceptualizar y redefinir el problema una y otra vez, integrando el trabajo con las siguientes fases secuenciales de acción: planificación, identificación de hechos, análisis, implementación y evaluación. Ver figura 1.

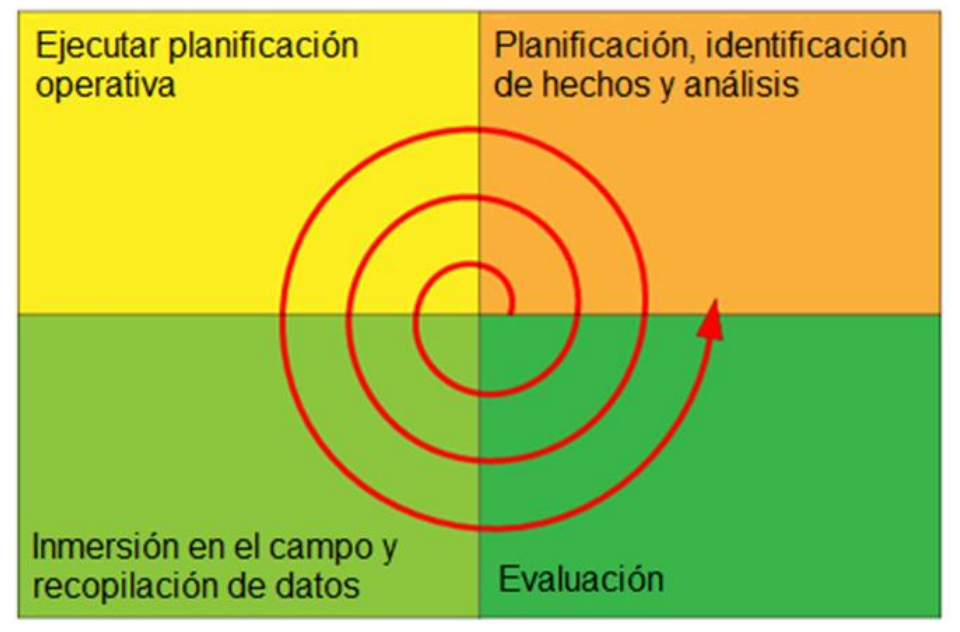

Ilustración 1: Método espiral de Investigación- Acción. Fuente: Hernández, 2010

En la primera fase de planificación se realizó un diagnóstico de los sitios en donde se presentaron los accidentes de tránsito durante el año 2014 en la ciudad de Loja, georreferenciando dicha información y registrando el tipo y la causa del accidente.

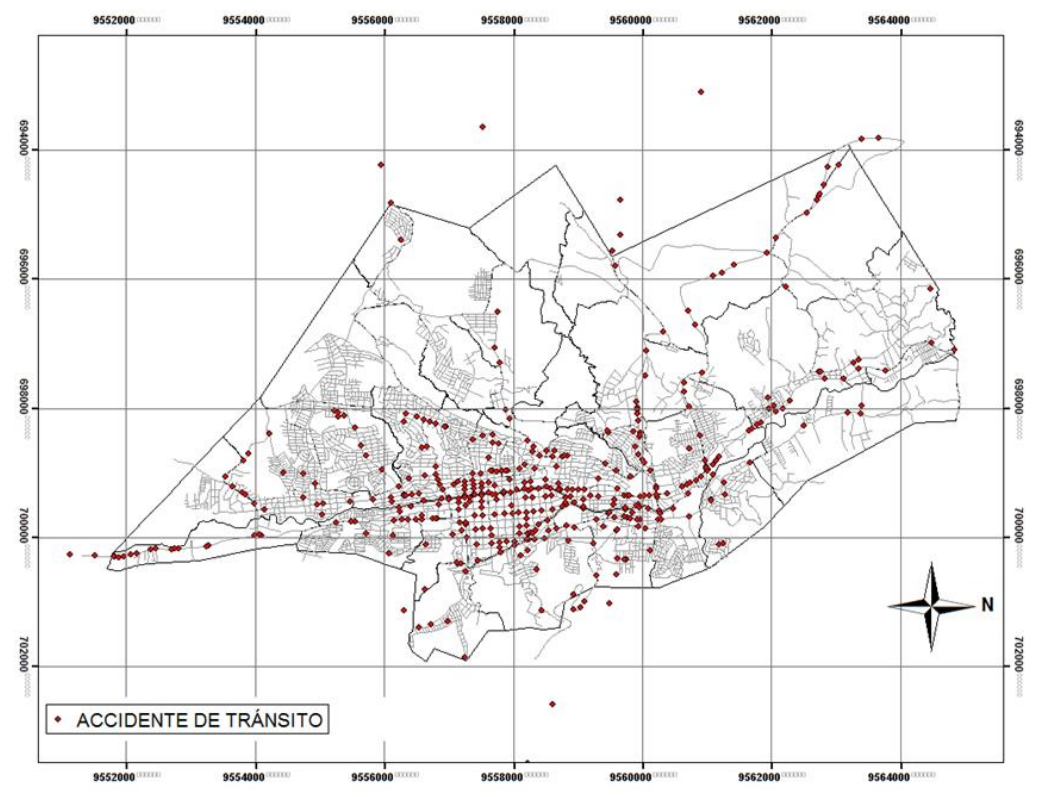


Ilustración 2: Ocurrencia de accidentes de tránsito en la ciudad de Loja en el año 2014. Fuente: Municipio de Loja, 2015

Con la información recabada se propuso la ejecución de una serie de actividades, entre ellas: Identificar sitios de mayor accidentabilidad, optimizar la programación operativa de la Policía Municipal de Tránsito, efectuar campañas de educación y seguridad vial dirigidas a la ciudadanía en general, implementar nuevos dispositivos de control de tránsito, capacitar y fortalecer con nueva logística a los policías municipales de tránsito, mejorar los procesos de revisión técnica vehicular, coordinar actividades de cooperación interinstitucional y concientizar a conductores de servicio de transporte público y comercial, todas estas actividades resumidas en un flexible plan de seguridad vial a ser ejecutado en el año 2015 y años posteriores.

Durante la ejecución de cada una de las actividades antes mencionadas se recopiló en una bitácora de campo toda la información estadística generada. El resultado del análisis de la información recabada permitió evaluar la efectividad de cada una de las actividades emprendidas en relación a la disminución del número de accidentes de tránsito y a la vez implementar las mejoras pertinentes en una nueva etapa de planificación, generando una nueva y mejorada versión del plan de seguridad vial, para en lo posterior ponerlo en la práctica, conduciendo el proceso de retroalimentación a un nuevo diagnóstico y a una nueva espiral de reflexión y acción.

\section{Resultados}

Durante todo el proceso investigativo se recopilaron datos generados a través de la aplicación de las diversas actividades contempladas en el plan de seguridad vial, relacionándola con la ocurrencia de accidentes de tránsito, lo que permitió revisar el material y organizarlo, emergiendo varias unidades de análisis que se agruparon en categorías por el método de comparación constante. De igual manera al comparar las categorías se las agrupó por temas, obteniendo un patrón de resultado como se muestra en la tabla 1.

Tabla 1: Estructuración y análisis de información de Campo.

\begin{tabular}{|c|c|c|}
\hline \multicolumn{3}{|c|}{ Estructuración Y Análisis De Información De Campo } \\
\hline Patrón & Tema & Categoría De Unidades De Análisis \\
\hline \multirow{14}{*}{$\begin{array}{l}\text { Ocurrencia de } \\
\text { accidentes de } \\
\text { tránsito en el } \\
\text { Cantón Loja }\end{array}$} & \multirow{9}{*}{$\begin{array}{l}\text { Formulación y ejecución de Planes } \\
\text { Operativos de control de Tránsito }\end{array}$} & Delitos de tránsito \\
\hline & & Contravenciones de primera clase \\
\hline & & Contravenciones de segunda clase \\
\hline & & Contravenciones de tercera clase \\
\hline & & Contravenciones de cuarta clase \\
\hline & & Contravenciones de quinta clase \\
\hline & & Contravenciones de sexta clase \\
\hline & & Contravenciones de séptima clase \\
\hline & & Procesos de juzgamiento \\
\hline & \multirow[t]{5}{*}{ Campaña de seguridad vial } & Brief de campaña \\
\hline & & $\begin{array}{l}\text { Socialización de campaña de seguridad vial a } \\
\text { instituciones públicas y privadas }\end{array}$ \\
\hline & & Apoyo de escuelas de conducción \\
\hline & & Actividades educativas en la vía pública \\
\hline & & Stand de Tránsito en la Feria de Loja \\
\hline
\end{tabular}




\begin{tabular}{|c|c|}
\hline & $\begin{array}{l}\text { Materiales visuales (Spot de Campaña, } \\
\text { animaciones, vallas, etc.) }\end{array}$ \\
\hline \multirow{10}{*}{$\begin{array}{l}\text { Capacitación y concientización a } \\
\text { conductores }\end{array}$} & Taxis convencionales \\
\hline & Taxis ejecutivos \\
\hline & Camionetas de alquiler \\
\hline & Transporte mixto de carga y pasajeros \\
\hline & Vehículos de transporte escolar \\
\hline & Volquetas de transporte de material pétreo \\
\hline & Buses de transporte público urbano \\
\hline & Buses de transporte público intracantonal \\
\hline & Buses de transporte público intraprovincial \\
\hline & $\begin{array}{l}\text { Buses de transporte público interprovincial e } \\
\text { internacional }\end{array}$ \\
\hline \multirow{5}{*}{$\begin{array}{l}\text { Capacitación a Policías Municipales de } \\
\text { Tránsito }\end{array}$} & Código Orgánico Integral Penal \\
\hline & Plan de Movilidad del cantón Loja \\
\hline & $\begin{array}{l}\text { Ley Orgánica de Transporte Terrestre, Tránsito } \\
\text { y Seguridad Vial }\end{array}$ \\
\hline & Sistema informático David \\
\hline & Relaciones humanas \\
\hline \multirow[t]{3}{*}{ Fortalecimiento de la policía de tránsito } & Proceso de selección de 100 nuevos aspirantes \\
\hline & $\begin{array}{l}\text { Curso de formación de } 100 \text { aspirantes } \\
\text { seleccionados }\end{array}$ \\
\hline & Proceso de adquisición de 24 motocicletas \\
\hline \multirow[t]{4}{*}{ Señalización y semaforización } & Señales verticales \\
\hline & Señales horizontales \\
\hline & Semáforos \\
\hline & Jerarquización vial \\
\hline \multirow[t]{3}{*}{ Matriculación vehicular } & Revisión técnica vehicular \\
\hline & Atención al público \\
\hline & Sistema informático de matriculación \\
\hline \multirow{8}{*}{$\begin{array}{l}\text { Revisión de los vehículos de transporte } \\
\text { público y comercial }\end{array}$} & Taxis convencionales \\
\hline & Taxis ejecutivos \\
\hline & Camionetas de alquiler \\
\hline & Transporte mixto de carga y pasajeros \\
\hline & Vehículos de transporte escolar \\
\hline & Volquetas de transporte de material pétreo \\
\hline & Buses de transporte público \\
\hline & Colocación de adhesivo municipal \\
\hline \multirow{3}{*}{$\begin{array}{l}\text { Operación de Terminal Terrestre Reina } \\
\text { del Cisne }\end{array}$} & Revisión de buses de transporte público \\
\hline & Control de conductores \\
\hline & Control de turnos y frecuencias \\
\hline \multirow[t]{7}{*}{ Procedimiento juzgador } & Jueces de tránsito \\
\hline & Fiscales \\
\hline & Impugnaciones \\
\hline & Sentencias \\
\hline & Policía Nacional SIAT \\
\hline & Personas detenidas \\
\hline & Vehículos retenidos \\
\hline
\end{tabular}




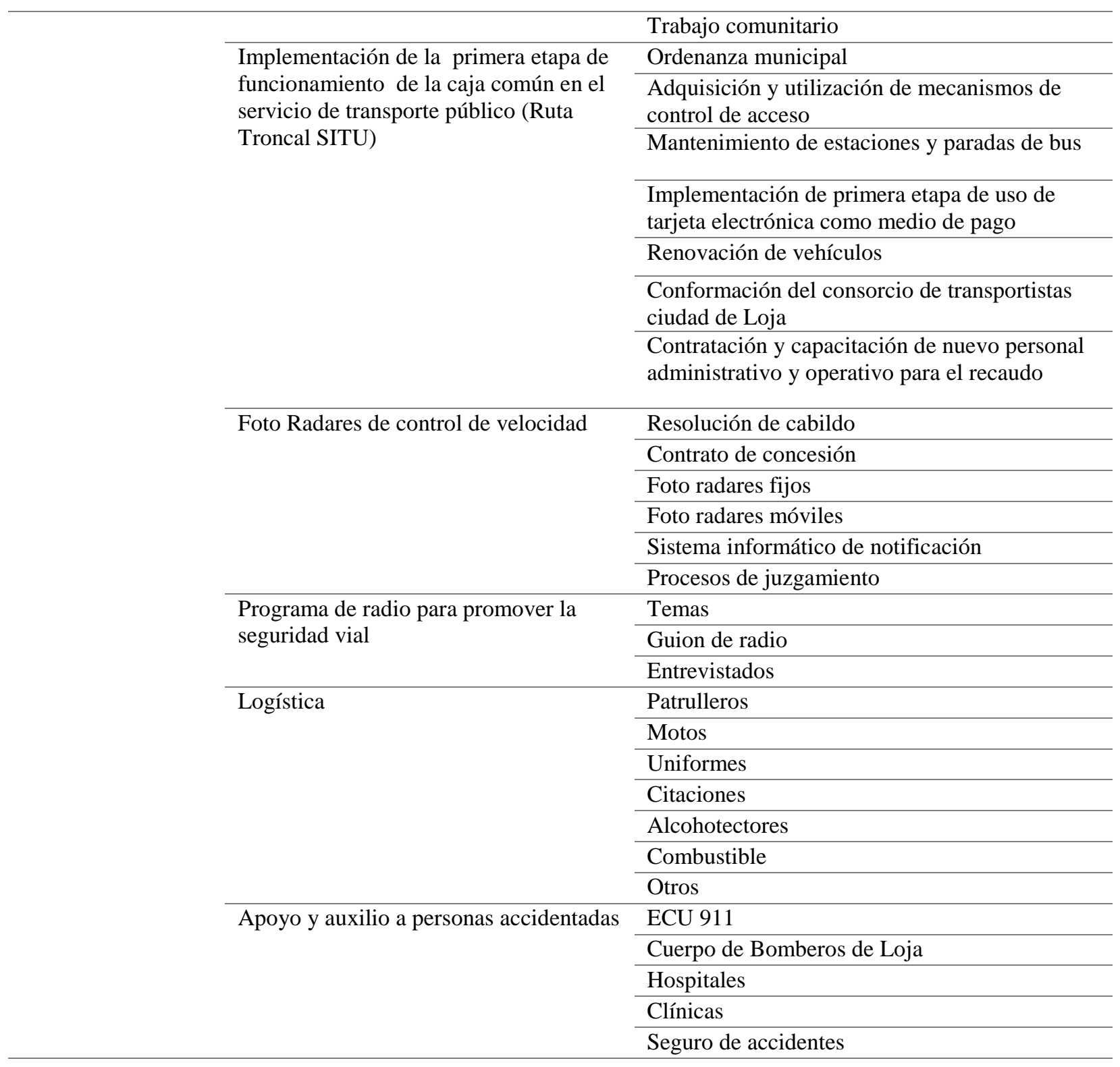

\section{Elaborado por: el autor}

Como resultado de la aplicación del método, en el año 2015 se presentaron 944 accidentes de tránsito $91 \%$ en el área urbana y $9 \%$ en el área rural, consiguiéndose con relación a lo ocurrido en el año 2014 una disminución de 772 accidentes, equivalente al 45\%, disminuyendo también en un $13 \%$ el número de personas fallecidas y en un $12 \%$ el número de personas heridas. Para el año 2016 se presentaron 526 accidentes de tránsito 87\% en el área urbana y $13 \%$ en el área rural, consiguiéndose con relación a lo ocurrido en el año 2015 una disminución de 418 accidentes, equivalente al 44\%, disminuyendo también en un $4 \%$ el número de personas fallecidas y en un 35\% el número de personas heridas. Ver Tabla 2.

Tabla 2: Accidentes de tránsito ocurridos en el año 2014, 2015 y 2016. 


\begin{tabular}{|c|c|c|c|}
\hline \multicolumn{4}{|c|}{ Accidentes De Tránsito Ocurridos En El Año 2014 Y 2015} \\
\hline \multirow[t]{2}{*}{ Mes } & \multicolumn{3}{|c|}{ Año } \\
\hline & 2014 & 2015 & 2016 \\
\hline Enero & 162 & 104 & 43 \\
\hline Febrero & 132 & 64 & 44 \\
\hline Marzo & 192 & 48 & 44 \\
\hline Abril & 162 & 83 & 49 \\
\hline Mayo & 181 & 95 & 51 \\
\hline Junio & 160 & 90 & 47 \\
\hline Julio & 103 & 98 & 49 \\
\hline Agosto & 136 & 62 & 42 \\
\hline Septiembre & 132 & 96 & 47 \\
\hline Octubre & 123 & 80 & 36 \\
\hline Noviembre & 99 & 47 & 31 \\
\hline Diciembre & 134 & 77 & 43 \\
\hline TOTAL & 1716 & 944 & 526 \\
\hline Fallecidos & 31 & 27 & 26 \\
\hline Heridos & 386 & 338 & 219 \\
\hline Población & 237160 & 243089 & 248072 \\
\hline Índice de accidentes por cada mil habitantes & 7.24 & 3.88 & 2.12 \\
\hline Índice de mortalidad por cada mil habitantes & 0.13 & 0.11 & 0.10 \\
\hline Índice de morbilidad por cada mil habitantes & 1.63 & 1.39 & 0.88 \\
\hline
\end{tabular}

Fuente: Municipio de Loja.

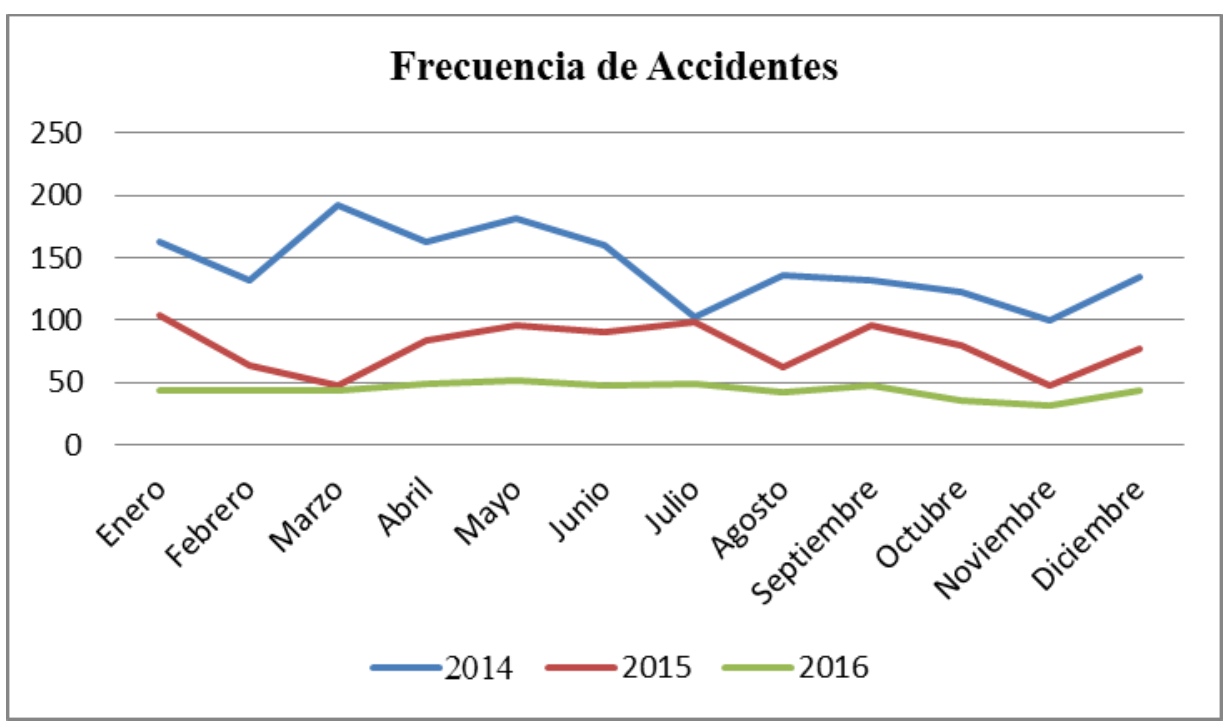

Elaborado por: el autor

De estos resultados, se puede apreciar la reducción de accidentes que ha habido en estos tres años, pero también se muestra una mayor ocurrencia de accidentes entre los meses de mayo y julio, como común denominador para los tres años analizados. De igual forma se puede 
observar que para el diagrama muestra una curva de frecuencia en el mes de diciembre para los tres años analizados.

En las tablas 3 y 4 se puede observar un comparativo de los tipos y causas de accidentes de tránsito ocurridos en el cantón Loja en el año 2014, 2015 y 2016. El porcentaje de accidentes producidos por causa humana fue del $96 \%$ en el 2015 y del $93 \%$ en el 2016.

Tabla 3: Tipos de accidentes de tránsito ocurridos en el año 2014, 2015 y 2016.

\begin{tabular}{|c|c|c|c|c|c|c|}
\hline \multicolumn{7}{|c|}{ Tipos De Accidentes De Tránsito Ocurridos En El Año 2014, 2015 Y 2016} \\
\hline \multirow[t]{2}{*}{ Tipo } & \multicolumn{2}{|c|}{2014} & \multicolumn{2}{|c|}{2015} & \multicolumn{2}{|c|}{2016} \\
\hline & $\begin{array}{c}\text { Nro. } \\
\text { Accidentes }\end{array}$ & $\%$ & $\begin{array}{c}\text { Nro. } \\
\text { Accidentes }\end{array}$ & $\%$ & $\begin{array}{c}\text { Nro. } \\
\text { Accidentes }\end{array}$ & $\%$ \\
\hline Atropello & 104 & $6.06 \%$ & 90 & $9.53 \%$ & 44 & $8.37 \%$ \\
\hline Arrollamiento & 4 & $0.23 \%$ & 3 & $0.32 \%$ & 1 & $0.19 \%$ \\
\hline Caída De Pasajeros & 11 & $0.64 \%$ & 10 & $1.06 \%$ & 3 & $0.57 \%$ \\
\hline Encunetamiento & 24 & $1.40 \%$ & 12 & $1.27 \%$ & 2 & $0.38 \%$ \\
\hline Estrellamiento & 288 & $16.78 \%$ & 173 & $18.33 \%$ & 143 & $27.19 \%$ \\
\hline Rozamiento & 216 & $12.59 \%$ & 74 & $7.84 \%$ & 24 & $4.56 \%$ \\
\hline Choque & 886 & $51.63 \%$ & 479 & $50.74 \%$ & 255 & $48.48 \%$ \\
\hline Colisión & 53 & $3.09 \%$ & 18 & $1.91 \%$ & 5 & $0.95 \%$ \\
\hline Volcamiento & 79 & $4.60 \%$ & 66 & $6.99 \%$ & 35 & $6.65 \%$ \\
\hline Perdida De Pista & 40 & $2.33 \%$ & 13 & $1.38 \%$ & 8 & $1.52 \%$ \\
\hline Otros & 11 & $0.64 \%$ & 6 & $0.64 \%$ & 6 & $1.14 \%$ \\
\hline Total & 1716 & $100.00 \%$ & 944 & $100.00 \%$ & 526 & $100.00 \%$ \\
\hline
\end{tabular}

Fuente: Municipio de Loja

Tabla 4: Causas de Accidentes de Tránsito ocurridos en el año 2014, 2015 y 2016.

\begin{tabular}{|c|c|c|c|c|c|c|}
\hline \multicolumn{7}{|c|}{ Causas De Accidentes De Tránsito Ocurridos En El Año 2014, 2015 Y 2016} \\
\hline \multirow[t]{2}{*}{ Causa } & \multicolumn{2}{|c|}{2014} & \multicolumn{2}{|c|}{2015} & \multicolumn{2}{|c|}{2016} \\
\hline & $\begin{array}{c}\text { Nro. } \\
\text { Accidentes }\end{array}$ & $\%$ & $\begin{array}{c}\text { Nro. } \\
\text { Accidentes }\end{array}$ & $\%$ & $\begin{array}{c}\text { Nro. } \\
\text { Accidentes }\end{array}$ & $\%$ \\
\hline Embriaguez Del Conductor & 175 & $10.20 \%$ & 150 & $15.89 \%$ & 134 & $25.48 \%$ \\
\hline Embriaguez Del Peatón & 3 & $0.17 \%$ & 3 & $0.32 \%$ & 1 & $0.19 \%$ \\
\hline $\begin{array}{l}\text { Impericia/Imprudencia Del } \\
\text { Conductor }\end{array}$ & 470 & $27.39 \%$ & 197 & $20.87 \%$ & 54 & $10.27 \%$ \\
\hline Exceso De Velocidad & 84 & $4.90 \%$ & 139 & $14.72 \%$ & 138 & $26.24 \%$ \\
\hline Negligencia Del Conductor & 722 & $42.07 \%$ & 302 & $31.99 \%$ & 79 & $15.02 \%$ \\
\hline Mal Rebasamiento & 23 & $1.34 \%$ & 23 & $2.44 \%$ & 23 & $4.37 \%$ \\
\hline Mal Estacionamiento & 3 & $0.17 \%$ & 3 & $0.32 \%$ & 0 & $0.00 \%$ \\
\hline Pasar Semáforo En Rojo & 20 & $1.17 \%$ & 17 & $1.80 \%$ & 21 & $3.99 \%$ \\
\hline Imprudencia Del Peatón & 47 & $2.74 \%$ & 40 & $4.24 \%$ & 26 & $4.94 \%$ \\
\hline Factores Climáticos & 11 & $0.64 \%$ & 5 & $0.53 \%$ & 2 & $0.38 \%$ \\
\hline
\end{tabular}




\begin{tabular}{ccccccc}
\hline Mal Estado De La Vía & 1 & $0.06 \%$ & 0 & $0.00 \%$ & 0 & $0.00 \%$ \\
\hline No Respeto De Señales & 18 & $1.05 \%$ & 4 & $0.42 \%$ & 5 & $0.95 \%$ \\
\hline Daños Mecánicos-Frenos & 19 & $1.11 \%$ & 15 & $1.59 \%$ & 20 & $3.80 \%$ \\
\hline Casos Fortuitos & 25 & $1.46 \%$ & 17 & $1.80 \%$ & 14 & $2.66 \%$ \\
\hline $\begin{array}{c}\text { Otras Causas (Por } \\
\text { Determinarse). }\end{array}$ & 76 & $4.43 \%$ & 26 & $2.75 \%$ & 8 & $1.52 \%$ \\
\hline Luminancia (Encandilamiento) & 0 & $0.00 \%$ & 1 & $0.11 \%$ & 0 & $0.00 \%$ \\
\hline Fallas De Iluminación & 2 & $0.12 \%$ & 0 & $0.00 \%$ & 0 & $0.00 \%$ \\
\hline Obstáculo En La Vía & 17 & $0.99 \%$ & 2 & $0.21 \%$ & 1 & $0.19 \%$ \\
\hline Total & 1716 & $100.00 \%$ & 944 & $100.00 \%$ & 526 & $100.00 \%$ \\
\hline
\end{tabular}

Fuente: Municipio de Loja. Elaborado por: el autor

De acuerdo a la gráfica de la ilustración 4; en el 2014 y 2015 la mayor causa de accidentes era la negligencia del conductor, en 2016 la mayor causa es el exceso de velocidad y embriaguez del conductor; en estos 3 años, los factores climáticos, obstáculo y estado de la vía han sido las causas menores frecuentes de accidentes de tránsito. Estos resultados hacen posible la evaluación de los métodos de la tabla 1, aplicados a los distintos temas del patrón de ocurrencia; comprobando su eficacia y generando un diagnóstico que facilita perfilar estrategias específicas a determinados temas de investigación en este campo.

Por otra parte, la evaluación por tema permite una lectura independiente de los métodos que han generado mayor impacto en la reducción de accidentes de tránsito, así por ejemplo en el tema "negligencia del conductor" se evidencia una reducción de esta causa al $10.0 \%$ con respecto al año 2014; así también en el tema de "impericia e imprudencia del conductor" se han reducido el $11.4 \%$ de accidentes por esta causa con respecto al año 2014. Sin embargo para temas específicos como "exceso de velocidad" el porcentaje de ocurrencias ha incrementado; en el año 2015 y 2016 las ocurrencias por esta causa son mayores de lo que fueron para el 2014.

\section{Discusión}

Los resultados del presente trabajo ratifican que los accidentes de tránsito no son cosa del azar, sino que pueden ser evitados; y por tanto las estrategias deben apuntar a la regulación y control, a través de la autoridad de tránsito, la cual debe promover de manera constante un ambiente seguro a favor de peatones, ciclistas, usuarios del transporte público y conductores de vehículos; además es necesario promover la educación vial y fortalecer los modos de transporte, para que sean cada vez más seguros, económicos, confortables y menos contaminantes.

El presente trabajo resalta la efectividad del método denominado investigación-acción expuesto en la literatura de Hernández (2010), el cual se aplica para mejorar prácticas concretas, como en este caso particular, en la promoción de la seguridad vial y disminución del número de accidentes de tránsito en el cantón Loja; además la evaluación de factores de manera independiente hace posible este método a temas específicos, de acuerdo a la evaluación de eficiencia de estrategias aplicadas a determinadas causa. El método puede ser replicado continuamente para desarrollar y mejorar prácticas o procesos institucionales, como por ejemplo los que ejecutan la Agencia Nacional de Tránsito, Municipios del Ecuador, Policía Nacional, Comisión de Tránsito del Ecuador, entre otros. 
Los resultados muestran que a pesar de tener una reducción de accidentes de tránsito, el patrón predominante cambia, poniendo el peso sobre "embriaguez del conductor" y "exceso de velocidad" como problemas principales para el año 2016, lo que permite cuestionar las estrategias vinculadas a pugnar estas causas y a relacionarlas con otros factores socioeconómicos simultáneos que podrían influir sobre estos resultados; planteando nuevas hipótesis y por tanto nuevas investigaciones.

Cabe mencionar que al ser los accidentes de tránsito, componentes de los costes externos del transporte (así como la contaminación y el ruido) que deben ser analizados desde la perspectiva de la movilidad sostenible (Lizárraga, 2006), es fundamental reconocer que estas estrategias deben vincularse a la situación general de la movilidad urbana, esto es, estudio de modalidades de transporte, localización y concentración de actividades, situación del transporte público y red vial entre otros factores, que también pueden beneficiarse de un diagnóstico de causas que generan la ocurrencia de accidentes de tránsito y su localización geográfica dentro de la ciudad.

\section{Conclusiones}

Se cumple afirmativamente la hipótesis, ya que la aplicación del método "investigación acción”, permitió a la UMTTTSV en el transcurso del año 2014, 2015 y 2016, ejecutar sistemáticamente una serie de actividades, cuyo producto fue la disminución de un $45 \%$ del número de accidentes de tránsito ocurridos en el año 2015 con relación al año 2014 y una reducción de 44\% de lo ocurrido en el año 2016 con relación al año 2015, superando lo planteado en la hipótesis.

Si consideramos los índices que relacionan la ocurrencia de accidentes de tránsito con el número habitantes en el cantón Loja, estos nos muestran para el año 2015 una reducción con relación a lo ocurrido en el año 2014 de un $46 \%$ en el índice de accidentalidad, $15 \%$ en el de mortalidad y $15 \%$ en el de morbilidad. Así mismo para el año 2016 se presenta una reducción con relación a lo ocurrido en el año 2015 de un 45\% en el índice de accidentalidad, 5\% en el de mortalidad y $36 \%$ en el de morbilidad. Estimando que la población se incrementó cada año en un $2.05 \%$ según las proyecciones del INEC.

El patrón predominante de causas que generan los accidentes de tránsito para la ciudad de Loja, pasaron a ser "exceso de velocidad" y "embriaguez del conductor" para el 2016, cuando en el 2015 habían sido "impericia" e "imprudencia" del conductor, lo cual abre nuevas hipótesis sobre la eficacia de la estrategia y sobre los factores socioeconómicos que podrían estar relacionados, influyendo sobre esta situación.

Los accidentes de tránsito son una de las externalidades negativas relacionadas a la movilidad urbana, por lo que deben ser analizadas paralelamente a otros factores relacionados a ella.

\section{Reconocimiento}


Se extiende un agradecimiento a la Universidad Internacional del Ecuador extensión Loja, en especial a la Arq. Tatiana Trokhimtchouk Directora del CipArq-Loja, por la motivación y las facilidades brindadas para elaborar el presente trabajo de investigación, por el tiempo invertido y los comentarios generados con respecto al tema, así mismo a funcionarios del Municipio de Loja específicamente a quienes laboran en la Unidad Municipal de Transporte Terrestre, Tránsito y Seguridad Vial.

\section{Bibliografía}

Asamblea Constituyente (2008). Constitución del Ecuador. Registro oficial del 20 de octubre de 2008. Ecuador.

Asamblea Nacional del Ecuador (2008). Ley Orgánica de Transporte Terrestre, Tránsito y Seguridad Vial. Registro Oficial Nro. 398 del 07 de agosto del 2008. Ecuador.

Asamblea Nacional del Ecuador (2010). Código Orgánico de Organización Territorial Autonomía y Descentralización. Registro Oficial Nro. 303 del 19 de octubre del 2010. Ecuador.

Asamblea Nacional del Ecuador (2014). Código Orgánico Integral Penal. Registro Oficial Nro. 180 del lunes 10 de febrero del 2014. Ecuador.

Hernández, R. (2010). Metodología de la Investigación. McGraw Hill, Quinta edición México. 613 pág.

Jiménez, C. (2015). Educación Vial “Hacia el tránsito seguro y sostenible”. España. McGraw Hill. Recuperado de: ProQuestebrary. Web.

Jorge Miguez, A., Godoy del Sol, H., \& Ortis Sagasta, M. (2010). Caracterización de la mortalidad por accidentes de tránsito con participación de ciclos. Medisur, 8(4), 274-279. Recuperado de http://www.medisur.sld.cu/index.php/medisur/article/view/1269/7181

Lizárraga, C. (2006). Movilidad urbana sostenible: un reto para las ciudades del siglo XXI. Economía, Sociedad y Territorio, vol. VI, núm. 22. Facultad de Ciencias Económicas y Empresariales, Universidad de Granada. España.

Municipio de Loja (2012). Plan de Movilidad del Cantón Loja 2012 - 2022. Ecuador.

Municipio de Loja (2014). Plan de Desarrollo y Ordenamiento Territorial del Cantón Loja 20142022. Ecuador.

Norza C., E. H., Granados L., E. L., Useche H., S. A., Romero H., M. \& Moreno R., J. (2014).

Componentes descriptivos y explicativos de la accidentalidad vial en Colombia: incidencia del factor humano. Revista Criminalidad, 56 (1): 157-187.

Toledo, F. y Montoro, L (1997). Drogas de abuso y Conducción. España. Editorial Intras. 
Tomas, A. (2004). Manual de seguridad Vial: El factor Humano. España. Editorial Ariel. Recuperado de: ProQuestebrary. Web. 